\title{
On Weighted Average Interpolation with Cardinal Splines
}

\author{
J. López-Salazar • G. Pérez-Villalón
}

\begin{abstract}
Given a sequence of data $\left\{y_{n}\right\}_{n \in \mathbb{Z}}$ with polynomial growth and an odd number $d$, Schoenberg proved that there exists a unique cardinal spline $f$ of degree $d$ with polynomial growth such that $f(n)=y_{n}$ for all $n \in \mathbb{Z}$. In this work, we show that this result also holds if we consider weighted average data $f * h(n)=y_{n}$, whenever the average function $h$ satisfies some light conditions. In particular, the interpolation result is valid if we consider cellaverage data $\int_{n-a}^{n+a} f(x) d x=y_{n}$ with $0<a \leq 1 / 2$. The case of even degree $d$ is also studied.
\end{abstract}

Keywords spline $\cdot$ cardinal interpolation $\cdot$ average sampling

\section{Introduction}

During the last forty years, the spaces of splines have become one of the most useful function spaces in applied mathematics. Within that area, this paper is devoted to the topic of interpolation with spline functions. Let $\beta_{d}$ be the central B-spline of degree $d \in \mathbb{N}$ given by

$$
\beta_{d}=\mathcal{X}_{[-1 / 2,1 / 2]} * \cdots * \mathcal{X}_{[-1 / 2,1 / 2]} \quad(d+1 \text { terms }),
$$

where $\mathcal{X}_{[-1 / 2,1 / 2]}$ denotes the characteristic function of the interval $[-1 / 2,1 / 2]$ and the symbol $*$ denotes the integral convolution. In this work we consider the space $\mathcal{S}_{d}$ generated by the integer shifts of the B-spline $\beta_{d}$. That is, a function $f$ belongs to $\mathcal{S}_{d}$ if and only if there is a unique sequence $\left\{a_{k}\right\}_{k \in \mathbb{Z}}$ in $\mathbb{C}$ such that

$$
f(x)=\sum_{k \in \mathbb{Z}} a_{k} \beta_{d}(x-k) .
$$


As is well-known, if $d$ is odd, then $\mathcal{S}_{d}$ coincides with the space of all functions $f \in C^{d-1}(\mathbb{R})$ such that $\left.f\right|_{[k, k+1]}$ is a polynomial of degree not exceeding $d$ for each $k \in \mathbb{Z}$. If $d$ is even, then $\mathcal{S}_{d}$ coincides with the space of all functions $f \in C^{d-1}(\mathbb{R})$ such that $\left.f\right|_{[k-1 / 2, k+1 / 2]}$ is a polynomial of degree not exceeding $d$ for every $k \in \mathbb{Z}$.

Given a sequence of real or complex numbers $\left\{y_{n}\right\}_{n \in \mathbb{Z}}$, there is a unique linear spline $f \in \mathcal{S}_{1}$ such that $f(n)=y_{n}$ for every $n \in \mathbb{Z}$, which is the function obtained by linear interpolation between every pair of consecutive data. On the contrary, for $d \geq 2$, there are infinitely many splines $f \in \mathcal{S}_{d}$ such that $f(n)=y_{n}$ for $n \in \mathbb{Z}$. However, Schoenberg proved in the seventies that if the function $f$ is required to have some growth conditions, then the interpolation problem has a unique solution, as the following theorem shows.

Theorem 1 (Shoenberg [9]) Let $\alpha \geq 0$. If $\left\{y_{n}\right\}_{n \in \mathbb{Z}}$ is a sequence in $\mathbb{C}$ such that $y_{n}=O\left(|n|^{\alpha}\right)$ as $n \rightarrow \pm \infty$, then there is a unique function $f \in \mathcal{S}_{d}$ such that $f(n)=y_{n}$ for all $n \in \mathbb{Z}$ and $f(x)=O\left(|x|^{\alpha}\right)$ as $x \rightarrow \pm \infty$.

Because of physical reasons, the available data often are not the values of a function $f$ at $n$, but weighted averages near $n$. That is,

$$
f * h(n)=\int_{-1 / 2}^{1 / 2} f(n-x) h(x) d x=\int_{n-1 / 2}^{n+1 / 2} f(x) h(n-x) d x,
$$

where the average function $h$, with support in $[-1 / 2,1 / 2]$, reflects the characteristic of the acquisition device. Note that $[-1 / 2,1 / 2]$ is the maximum possible support of $h$ without overlap between the samples. The average interpolation problem $f * h(n)=y_{n}$ has been studied for band-limited functions and for shift invariant spaces in [1-8, 11, 12] and [13].

The aim of this paper is to prove the following theorem.

Theorem 2 Let $h: \mathbb{R} \rightarrow \mathbb{R}$ be a measurable function that satisfies the following properties:

(a) $h(x) \geq 0$ for all $x \in \mathbb{R}$.

(b) The support of $h$ is contained in $[-1 / 2,1 / 2]$.

(c) $0<\int_{-1 / 2}^{0} h(x) d x<\infty$ and $0<\int_{0}^{1 / 2} h(x) d x<\infty$.

Let $d \in \mathbb{N}$ and $\alpha \geq 0$. If $\left\{y_{n}\right\}_{n \in \mathbb{Z}}$ is a sequence in $\mathbb{C}$ such that $y_{n}=O\left(|n|^{\alpha}\right)$ as $n \rightarrow \pm \infty$, then there is a unique function $f \in \mathcal{S}_{d}$ such that $f * h(n)=y_{n}$ for all $n \in \mathbb{Z}$ and $f(x)=$ $O\left(|x|^{\alpha}\right)$ as $x \rightarrow \pm \infty$.

Theorem 2 was proven in [5] for degree $d=1,2,3,4$. In [6] and [7], it was proved without limitation on the degree, but with more restrictive conditions on the average function $h$.

Our approach to Theorem 2 is based on the following result.

Theorem 3 (Pérez and Portal [5]) Let $d \in \mathbb{N}$ and let $h$ be a function with the properties given in Theorem 2. Let us assume that all the zeros of the function

$$
G(t)=\sum_{k \in \mathbb{Z}}\left[\beta_{d} * h(k)\right] t^{-k}
$$

are simple and none of them is on the unit circle $\{z \in \mathbb{C}:|z|=1\}$. If $\alpha \geq 0$ and $\left\{y_{n}\right\}_{n \in \mathbb{Z}}$ is $a$ sequence in $\mathbb{C}$ such that $y_{n}=O\left(|n|^{\alpha}\right)$ as $n \rightarrow \pm \infty$, then there is a unique function $f \in \mathcal{S}_{d}$ such that $f * h(n)=y_{n}$ for all $n \in \mathbb{Z}$ and $f(x)=O\left(|x|^{\alpha}\right)$ as $x \rightarrow \pm \infty$. 
In order to apply Theorem 3, all the results presented below will be used to prove that the zeros of $G$ satisfy the required conditions.

After this paper was finished, the authors knew that the same result had been obtained independently and at the same time by Ponnaian and Shanmugam in [8]. Although both papers use Theorem 3, the way those authors follow to study the zeros of the $G$ function is different from ours. Ponnaian and Shanmugam base their arguments on the study that they carry out about the roots of the exponential Euler splines. In particular, they need to obtain a recursion relation for those exponential splines. Moreover, they consider four different cases on the degree $d$. On the contrary, our arguments may be more direct, since we apply already known properties of the Euler-Frobenius polynomials and only have to consider even and odd degree cases separately.

\section{The Roots of $G$ when $d$ Is Even}

The following lemma gives the degree of the Laurent polynomial $G$.

Lemma 1 Let $h$ be a function with the properties given in Theorem 2. For $d \in \mathbb{N}$ and $k \in \mathbb{Z}$, it holds

(i) $\beta_{d} * h(k)=0$ if $|k| \geq \frac{d+2}{2}$.

(ii) $\beta_{d} * h(k)>0$ if $|k| \leq \frac{d+1}{2}$.

Proof Let us recall that $\beta_{d}$ is a continuous function such that $\beta_{d}(x)>0$ if $-\frac{d+1}{2}<x<\frac{d+1}{2}$ and $\beta_{d}(x)=0$ if $x \notin\left(-\frac{d+1}{2}, \frac{d+1}{2}\right)$. The support of $h$ is contained in $[-1 / 2,1 / 2]$, so

$$
\beta_{d} * h(k)=\int_{-1 / 2}^{1 / 2} \beta_{d}(k-x) h(x) d x .
$$

If $|k| \geq \frac{d+2}{2}$ and $x \in[-1 / 2,1 / 2]$, then $\beta_{d}(k-x)=0$, so $\beta_{d} * h(k)=0$.

Since $\int_{0}^{1 / 2} h(x) d x>0$, there is $\varepsilon \in(0,1 / 2)$ such that $\int_{\varepsilon}^{1 / 2} h(x) d x>0$. Let

$$
M=\min \left\{\beta_{d}(x):-\frac{1}{2} \leq x \leq \frac{d+1}{2}-\varepsilon\right\}>0 .
$$

If $0 \leq k \leq \frac{d+1}{2}$ and $x \in[\varepsilon, 1 / 2]$, then $k-x \in\left[-\frac{1}{2}, \frac{d+1}{2}-\varepsilon\right]$. Hence

$$
\beta_{d} * h(k) \geq \int_{\varepsilon}^{1 / 2} \beta_{d}(k-x) h(x) d x \geq M \cdot \int_{\varepsilon}^{1 / 2} h(x) d x>0 .
$$

Similarly, using that $\int_{-1 / 2}^{0} h(x) d x>0$, we obtain that $\beta_{d} * h(k)>0$ if $-\frac{d+1}{2} \leq k \leq 0$.

Let us now define the splines $\Upsilon_{t, d}$ that will be extensively used throughout the paper. For each $t \in \mathbb{C} \backslash\{0\}$ and each $d \in \mathbb{N}$, the symbol $\Upsilon_{t, d}$ denotes the function

$$
\Upsilon_{t, d}(x)=\sum_{k \in \mathbb{Z}} t^{-k} \beta_{d}(x-k) .
$$

It is easy to check that if $x \in \mathbb{R}$ and $n \in \mathbb{Z}$, then

$$
\Upsilon_{t, d}(x+n)=t^{-n} \Upsilon_{t, d}(x), \quad \Upsilon_{t^{-1}, d}(-x)=\Upsilon_{t, d}(x)
$$


Moreover, if $d \geq 2$, then

$$
\Upsilon_{t, d}^{\prime}(x)=(1-t) \Upsilon_{t, d-1}(x+1 / 2)
$$

Property (2) can be deduced from the following known fact:

$$
\beta_{d}^{\prime}(x)=\beta_{d-1}(x+1 / 2)-\beta_{d-1}(x-1 / 2) .
$$

Lemma 2 For any $t<0$ and any $d \in \mathbb{N}$, the function $\Upsilon_{t, d}$ has a unique root in $[-1 / 2,1 / 2)$.

Proof By (1), $\Upsilon_{t, d}(1 / 2)=t^{-1} \Upsilon_{t, d}(-1 / 2)$. Since $t<0$, there are two possibilities:

$$
\left\{\begin{array}{l}
\Upsilon_{t, d}(-1 / 2)=\Upsilon_{t, d}(1 / 2)=0 \\
\text { or } \\
\Upsilon_{t, d}(-1 / 2) \text { and } \Upsilon_{t, d}(1 / 2) \text { have different sign. }
\end{array}\right.
$$

The case $d=1$ can be easily deduced in any of the above possibilities, having in mind that $\Upsilon_{t, 1}(x)$ is a piecewise linear spline with knots in $\mathbb{Z}$ and $\Upsilon_{t, 1}(0)=1$.

We now assume that the result holds for some $d \in \mathbb{N}$; that is, there is a unique $a_{t, d} \in$ $[-1 / 2,1 / 2)$ such that $\Upsilon_{t, d}\left(a_{t, d}\right)=0$. The spline $\Upsilon_{t, d+1}$ is a $C^{d}$-function on $\mathbb{R}$. By (2),

$$
\Upsilon_{t, d+1}^{\prime}(x)=(1-t) \Upsilon_{t, d}(x+1 / 2)=(1-t) t^{-1} \Upsilon_{t, d}(x-1 / 2) .
$$

If $\Upsilon_{t, d+1}$ has a local extremum at a point $x_{0} \in(-1 / 2,1 / 2)$, then $\Upsilon_{t, d+1}^{\prime}\left(x_{0}\right)=0$, so $\Upsilon_{t, d}\left(x_{0}+\right.$ $1 / 2)=\Upsilon_{t, d}\left(x_{0}-1 / 2\right)=0$. Consequently, $\Upsilon_{t, d+1}$ has at most one local extremum $x_{0}$ on the interval $(-1 / 2,1 / 2)$ :

$$
\left\{\begin{array}{l}
x_{0}=a_{t, d}-1 / 2 \quad \text { if } a_{t, d} \in(0,1 / 2) \\
\text { or } \\
x_{0}=a_{t, d}+1 / 2 \quad \text { if } a_{t, d} \in[-1 / 2,0) .
\end{array}\right.
$$

That implies the following consequences:

1. If $\Upsilon_{t, d+1}(-1 / 2)=\Upsilon_{t, d+1}(1 / 2)=0$, then $\Upsilon_{t, d+1}(x)>0$ for every $x \in(-1 / 2,1 / 2)$ or $\Upsilon_{t, d+1}(x)<0$ for every $x \in(-1 / 2,1 / 2)$. In this case, $-1 / 2$ is the unique root of $\Upsilon_{t, d+1}$ on $[-1 / 2,1 / 2)$.

2. If $\Upsilon_{t, d+1}(-1 / 2)$ and $\Upsilon_{t, d+1}(1 / 2)$ have different sign, then there is $a_{t, d+1} \in(-1 / 2,1 / 2)$ such that $\Upsilon_{t, d+1}\left(a_{t, d+1}\right)=0$. As $\Upsilon_{t, d+1}$ has at most one local extremum on $(-1 / 2,1 / 2)$, it follows that $\Upsilon_{t, d+1}(x) \neq 0$ for every $x \in[-1 / 2,1 / 2), x \neq a_{t, d+1}$.

This concludes the proof for $d+1$, so the result holds for every $d \in \mathbb{N}$.

In the proof of the following lemmas we use the Euler-Frobenius polynomials, which are defined in terms of the forward B-splines

$$
Q_{d+1}(x)=\beta_{d}\left(x-\frac{d+1}{2}\right) .
$$

Here $Q_{d+1}(x) \neq 0$ if and only if $x \in(0, d+1)$. The Euler-Frobenius polynomial of degree $d-1$ is the function

$$
\Pi_{d}(t)=d ! \sum_{k=0}^{d-1} Q_{d+1}(k+1) t^{k}
$$


Then $\Pi_{d}$ is a monic polynomial of degree $d-1$ whose roots are all simple and negative. If $\lambda_{1}<\cdots<\lambda_{d-1}$ are the roots of $\Pi_{d}$ and $\mu_{1}<\cdots<\mu_{d-2}$ are the roots of $\Pi_{d-1}$, then

$$
\lambda_{1}<\mu_{1}<\lambda_{2}<\mu_{2}<\lambda_{3}<\cdots<\lambda_{d-2}<\mu_{d-2}<\lambda_{d-1} .
$$

Moreover, $\lambda_{1} \lambda_{d-1}=\lambda_{2} \lambda_{d-2}=\cdots=1$, so if $d$ is even, then

$$
\lambda_{d / 2}=-1
$$

For the proof of these facts, see Schoenberg [10, pp. 391-392].

Lemma 3 Let $d \in \mathbb{N}$ be even and let $\lambda_{1}<\cdots<\lambda_{d-1}$ be the roots of $\Pi_{d}$. Then

$$
\operatorname{sign}\left(\Upsilon_{\lambda_{j}, d}(x)\right)=(-1)^{j+\frac{d}{2}}
$$

for every $x \in(-1 / 2,1 / 2)$ and every $j \in\{1, \ldots, d-1\}$.

Proof Let $\lambda$ be any root of $\Pi_{d}$. Then

$$
\begin{aligned}
\Upsilon_{\lambda, d}(-1 / 2) & =\sum_{k \in \mathbb{Z}} \lambda^{-k} \beta_{d}\left(-\frac{1}{2}-k\right)=\sum_{k \in \mathbb{Z}} \lambda^{k} Q_{d+1}\left(k+\frac{d}{2}\right) \\
& =\sum_{k \in \mathbb{Z}} \lambda^{k+1-\frac{d}{2}} Q_{d+1}(k+1)=\frac{\lambda^{1-\frac{d}{2}}}{d !} \Pi_{d}(\lambda)=0 .
\end{aligned}
$$

By Lemma 2, $\Upsilon_{\lambda, d}$ only has one root on $[-1 / 2,1 / 2)$, so the sign of $\Upsilon_{\lambda, d}$ is constant on $(-1 / 2,1 / 2)$. Since $\Upsilon_{\lambda, d}(-1 / 2)=0$, we obtain the following alternatives:

$$
\begin{cases}\Upsilon_{\lambda, d}(x)>0 \text { for all } x \in(-1 / 2,1 / 2) & \text { if } \Upsilon_{\lambda, d}^{\prime}(-1 / 2)>0 \\ \text { or } & \text { if } \Upsilon_{\lambda, d}^{\prime}(-1 / 2)<0 \\ \Upsilon_{\lambda, d}(x)<0 \text { for all } x \in(-1 / 2,1 / 2)\end{cases}
$$

We now study the sign of $\Upsilon_{\lambda, d}^{\prime}(-1 / 2)$. By (2),

$$
\begin{aligned}
\Upsilon_{\lambda, d}^{\prime}(-1 / 2) & =(1-\lambda) \Upsilon_{\lambda, d-1}(0)=(1-\lambda) \sum_{k \in \mathbb{Z}} \lambda^{k} \beta_{d-1}(k) \\
& =(1-\lambda) \sum_{k \in \mathbb{Z}} \lambda^{k} Q_{d}\left(k+\frac{d}{2}\right)=(1-\lambda) \sum_{k \in \mathbb{Z}} \lambda^{k+1-\frac{d}{2}} Q_{d}(k+1) \\
& =(1-\lambda) \lambda^{1-\frac{d}{2}} \sum_{k=0}^{d-2} \lambda^{k} Q_{d}(k+1)=\frac{(1-\lambda)}{(d-1) !} \lambda^{1-\frac{d}{2}} \cdot \Pi_{d-1}(\lambda)
\end{aligned}
$$

Since $\lambda<0$ and $x \in(-1 / 2,1 / 2)$, we have

$$
\operatorname{sign}\left(\Upsilon_{\lambda, d}(x)\right)=\operatorname{sign}\left(\Upsilon_{\lambda, d}^{\prime}(-1 / 2)\right)=(-1)^{1-\frac{d}{2}} \cdot \operatorname{sign}\left(\Pi_{d-1}(\lambda)\right) .
$$

This study holds when $\lambda$ is any root of $\Pi_{d}$. 
Let us first assume that $d=2$. Since $\Pi_{1}(t)=1$ for every $t \in \mathbb{R}$, we have

$$
\operatorname{sign}\left(\Upsilon_{\lambda, 2}(x)\right)=(-1)^{1-\frac{2}{2}} \cdot \operatorname{sign}\left(\Pi_{1}(\lambda)\right)=1 .
$$

for all $x \in(-1 / 2,1 / 2)$. That proves the statement of the Lemma when $d=2$.

If $d$ is an even integer bigger than $2, \lambda_{1}<\cdots<\lambda_{d-1}$ are the roots of $\Pi_{d}$ and $\mu_{1}<\cdots<$ $\mu_{d-2}$ are the roots of $\Pi_{d-1}$, then

$$
\lambda_{1}<\mu_{1}<\lambda_{2}<\mu_{2}<\lambda_{3}<\cdots<\lambda_{d-2}<\mu_{d-2}<\lambda_{d-1} .
$$

As $\Pi_{d-1}$ is monic and its roots are simple, it can be written as

$$
\Pi_{d-1}(t)=\left(t-\mu_{1}\right)\left(t-\mu_{2}\right) \cdots\left(t-\mu_{d-2}\right)
$$

Then $\Pi_{d-1}\left(\lambda_{1}\right)>0, \Pi_{d-1}\left(\lambda_{2}\right)<0, \ldots \Pi_{d-1}\left(\lambda_{d-1}\right)>0$. That is,

$$
\operatorname{sign}\left(\Pi_{d-1}\left(\lambda_{j}\right)\right)=(-1)^{j+1}
$$

for every $j=1, \ldots, d-1$. Therefore, if $x \in(-1 / 2,1 / 2)$, then

$$
\begin{aligned}
\operatorname{sign}\left(\Upsilon_{\lambda_{j}, d}(x)\right) & =(-1)^{1-\frac{d}{2}} \cdot \operatorname{sign}\left(\Pi_{d-1}\left(\lambda_{j}\right)\right) \\
& =(-1)^{1-\frac{d}{2}} \cdot(-1)^{j+1}=(-1)^{j+\frac{d}{2}} .
\end{aligned}
$$

This completes the proof.

Theorem 4 Let $h$ be a function with the properties given in Theorem 2. If $d \in \mathbb{N}$ is even, then the function

$$
G(t)=\sum_{k \in \mathbb{Z}}\left[\beta_{d} * h(k)\right] t^{-k}
$$

has $d$ roots which are simple, negative and different from -1 .

Proof We will study the roots of the function

$$
F(t)=t^{d / 2} G(t)=\sum_{k \in \mathbb{Z}}\left[\beta_{d} * h(k)\right] t^{\frac{d}{2}-k}
$$

By Lemma $1, F$ is a polynomial of degree $d$ :

$$
F(t)=\beta_{d} * h\left(-\frac{d}{2}\right) t^{d}+\cdots+\beta_{d} * h\left(\frac{d}{2}-1\right) t+\beta_{d} * h\left(\frac{d}{2}\right) .
$$

Since the coefficients of $F$ are all positive, we have $F(0)>0$ and $\lim _{t \rightarrow-\infty} F(t)=+\infty$. Thus, $F$ and $G$ have exactly the same roots.

The function $\beta_{d}$ is even, so if $t \neq 0$, then

$$
F(t)=t^{d / 2} \sum_{k \in \mathbb{Z}} t^{-k} \int_{-1 / 2}^{1 / 2} \beta_{d}(k-x) h(x) d x=\int_{-1 / 2}^{1 / 2} t^{d / 2} \Upsilon_{t, d}(x) h(x) d x
$$


Let $\lambda_{1}<\cdots<\lambda_{d-1}$ be the roots of $\Pi_{d}$, which are all negative. By Lemma 3,

$$
\operatorname{sign}\left(\lambda_{j}^{d / 2} \Upsilon_{\lambda_{j}, d}(x)\right)=(-1)^{d / 2} \cdot(-1)^{j+\frac{d}{2}}=(-1)^{j}
$$

for every $x \in(-1 / 2,1 / 2)$ and every $j \in\{1, \ldots, d-1\}$. By (4) and (5) and having in mind that $h$ is non-negative, we have

$$
F\left(\lambda_{1}\right)<0, \quad F\left(\lambda_{2}\right)>0, \quad \ldots \quad F\left(\lambda_{d-1}\right)<0 .
$$

Since $\lim _{t \rightarrow-\infty} F(t)=+\infty$ and $F(0)>0$, there are

$$
s_{1} \in\left(-\infty, \lambda_{1}\right), s_{2} \in\left(\lambda_{1}, \lambda_{2}\right), \ldots, s_{d-1} \in\left(\lambda_{d-2}, \lambda_{d-1}\right), s_{d} \in\left(\lambda_{d-1}, 0\right)
$$

such that $F\left(s_{1}\right)=0, \ldots, F\left(s_{d}\right)=0$. As $F$ is a polynomial of degree $d$, it cannot have other roots. By (3), it is known that $\lambda_{d / 2}=-1$, so $F(-1) \neq 0$. Therefore, $s_{1} \neq-1, \ldots, s_{d} \neq$ -1 .

Remark 1 Theorem 2 with $d$ even is now a direct consequence of Theorems 3 and 4 .

\section{The Roots of $G$ when $d$ Is Odd}

In order to study the roots of $G$ when $d$ is odd, we use the midpoint Euler-Frobenius polynomials introduced by Schoenberg [10, pp. 393-394]. Given $d \in \mathbb{N}$, the function

$$
P_{d}(t)=2^{d} d ! \sum_{k=0}^{d} Q_{d+1}\left(k+\frac{1}{2}\right) t^{k}
$$

is a monic polynomial of degree $d$ whose roots are all simple and negative. If $\lambda_{1}<\cdots<\lambda_{d}$ are the roots of $P_{d}$ and $\mu_{1}<\cdots<\mu_{d-1}$ are the roots of $P_{d-1}$, then

$$
\lambda_{1}<\mu_{1}<\lambda_{2}<\mu_{2}<\lambda_{3}<\cdots<\lambda_{d-1}<\mu_{d-1}<\lambda_{d} .
$$

Moreover, if $d$ is odd, then

$$
\lambda_{(d+1) / 2}=-1
$$

Lemma 4 Let $d \in \mathbb{N}$ be odd and let $\lambda_{1}<\cdots<\lambda_{d}$ be the roots of $P_{d}$. Then

$$
\operatorname{sign}\left(\Upsilon_{\lambda_{j}, d}(x)\right)=(-1)^{j+\frac{d+1}{2}}
$$

for every $x \in(-1 / 2,1 / 2)$ and every $j \in\{1, \ldots, d\}$.

Proof Let $\lambda$ be any root of $P_{d}$. Then

$$
\begin{aligned}
\Upsilon_{\lambda, d}\left(-\frac{1}{2}\right) & =\sum_{k \in \mathbb{Z}} \lambda^{-k} \beta_{d}\left(-\frac{1}{2}-k\right)=\sum_{k \in \mathbb{Z}} \lambda^{k} Q_{d+1}\left(k+\frac{d}{2}\right) \\
& =\sum_{k \in \mathbb{Z}} \lambda^{k-\frac{d-1}{2}} Q_{d+1}\left(k+\frac{1}{2}\right)=\frac{\lambda^{\frac{1-d}{2}}}{2^{d} d !} P_{d}(\lambda)=0 .
\end{aligned}
$$


By Lemma 2, $\Upsilon_{\lambda, d}$ only has one root on $[-1 / 2,1 / 2)$, so the sign of $\Upsilon_{\lambda, d}$ is constant on $(-1 / 2,1 / 2)$.

If $d=1$, then

$$
\Upsilon_{\lambda, 1}(0)=\sum_{k \in \mathbb{Z}} \lambda^{-k} \beta_{1}(-k)=1
$$

Therefore, $\operatorname{sign}\left(\Upsilon_{\lambda, 1}(x)\right)=1$ for every $x \in(-1 / 2,1 / 2)$. That proves the statement of the Lemma when $d=1$.

Let us now assume that $d$ is any odd integer bigger than or equal to 3 . Since $\Upsilon_{\lambda, d}(-1 / 2)=0$ and the sign of $\Upsilon_{\lambda, d}$ is constant on $(-1 / 2,1 / 2)$, we obtain the following alternatives:

$$
\begin{cases}\Upsilon_{\lambda, d}(x)>0 \text { for all } x \in(-1 / 2,1 / 2) & \text { if } \Upsilon_{\lambda, d}^{\prime}(-1 / 2)>0 \\ \text { or } & \\ \Upsilon_{\lambda, d}(x)<0 \text { for all } x \in(-1 / 2,1 / 2) & \text { if } \Upsilon_{\lambda, d}^{\prime}(-1 / 2)<0\end{cases}
$$

We now study the sign of $\Upsilon_{\lambda, d}^{\prime}(-1 / 2)$. By (2),

$$
\begin{aligned}
\Upsilon_{\lambda, d}^{\prime}\left(-\frac{1}{2}\right) & =(1-\lambda) \Upsilon_{\lambda, d-1}(0)=(1-\lambda) \sum_{k \in \mathbb{Z}} \lambda^{k} \beta_{d-1}(k) \\
& =(1-\lambda) \sum_{k \in \mathbb{Z}} \lambda^{k} Q_{d}\left(k+\frac{d}{2}\right)=(1-\lambda) \sum_{k \in \mathbb{Z}} \lambda^{k-\frac{d-1}{2}} Q_{d}\left(k+\frac{1}{2}\right) \\
& =(1-\lambda) \lambda^{\frac{1-d}{2}} \sum_{k=0}^{d-1} \lambda^{k} Q_{d}\left(k+\frac{1}{2}\right)=\frac{(1-\lambda)}{2^{d-1}(d-1) !} \lambda^{\frac{1-d}{2}} \cdot P_{d-1}(\lambda)
\end{aligned}
$$

Since $\lambda<0$ and $x \in(-1 / 2,1 / 2)$, we have

$$
\operatorname{sign}\left(\Upsilon_{\lambda, d}(x)\right)=\operatorname{sign}\left(\Upsilon_{\lambda, d}^{\prime}(-1 / 2)\right)=(-1)^{\frac{1-d}{2}} \cdot \operatorname{sign}\left(P_{d-1}(\lambda)\right) .
$$

This study holds when $\lambda$ is any root of $P_{d}$.

If $\lambda_{1}<\cdots<\lambda_{d}$ are the roots of $P_{d}$ and $\mu_{1}<\cdots<\mu_{d-1}$ are the roots of $P_{d-1}$, then

$$
\lambda_{1}<\mu_{1}<\lambda_{2}<\mu_{2}<\lambda_{3}<\cdots<\lambda_{d-1}<\mu_{d-1}<\lambda_{d} .
$$

As $P_{d-1}$ is monic and its roots are simple, it can be written as

$$
P_{d-1}(t)=\left(t-\mu_{1}\right)\left(t-\mu_{2}\right) \cdots\left(t-\mu_{d-1}\right) .
$$

Then $P_{d-1}\left(\lambda_{1}\right)>0, P_{d-1}\left(\lambda_{2}\right)<0, \ldots P_{d-1}\left(\lambda_{d}\right)>0$. That is,

$$
\operatorname{sign}\left(P_{d-1}\left(\lambda_{j}\right)\right)=(-1)^{j+1}
$$

for every $j=1, \ldots, d$. Therefore, if $x \in(-1 / 2,1 / 2)$, then

$$
\begin{aligned}
\operatorname{sign}\left(\Upsilon_{\lambda_{j}, d}(x)\right) & =(-1)^{\frac{1-d}{2}} \cdot \operatorname{sign}\left(P_{d-1}\left(\lambda_{j}\right)\right) \\
& =(-1)^{\frac{1-d}{2}} \cdot(-1)^{j+1}=(-1)^{j+\frac{d+1}{2}}
\end{aligned}
$$

This completes the proof. 
Theorem 5 Let $h$ be a function with the properties given in Theorem 2. If $d \in \mathbb{N}$ is odd, then the function

$$
G(t)=\sum_{k \in \mathbb{Z}}\left[\beta_{d} * h(k)\right] t^{-k}
$$

has $d+1$ roots which are simple, negative and different from -1 .

Proof We will study the roots of the function

$$
H(t)=t^{\frac{d+1}{2}} G(t)=\sum_{k \in \mathbb{Z}}\left[\beta_{d} * h(k)\right] t^{\frac{d+1}{2}-k} .
$$

By Lemma $1, H$ is a polynomial of degree $d+1$ :

$$
H(t)=\beta_{d} * h\left(-\frac{d+1}{2}\right) t^{d+1}+\cdots+\beta_{d} * h\left(\frac{d+1}{2}-1\right) t+\beta_{d} * h\left(\frac{d+1}{2}\right) .
$$

Again the coefficients of $H$ are all positive, which yields $H(0)>0$ and $\lim _{t \rightarrow-\infty} H(t)=$ $+\infty$. Hence, all zeros of $H$ and $G$ coincide.

As in (4), if $t \neq 0$, then

$$
H(t)=\int_{-1 / 2}^{1 / 2} t^{\frac{d+1}{2}} \Upsilon_{t, d}(x) h(x) d x .
$$

Let $\lambda_{1}<\cdots<\lambda_{d}$ be the roots of the $P_{d}$, which are all negative. By Lemma 4,

$$
\operatorname{sign}\left(\left(\lambda_{j}\right)^{\frac{d+1}{2}} \Upsilon_{\lambda_{j}, d}(x)\right)=(-1)^{\frac{d+1}{2}}(-1)^{j+\frac{d+1}{2}}=(-1)^{j}
$$

for every $x \in(-1 / 2,1 / 2)$ and every $j \in\{1, \ldots, d\}$. By (7) and (8),

$$
H\left(\lambda_{1}\right)<0, \quad H\left(\lambda_{2}\right)>0, \quad \ldots \quad H\left(\lambda_{d}\right)<0 .
$$

Since $H(0)>0$ and $\lim _{t \rightarrow-\infty} H(t)=+\infty$, there are

$$
s_{1} \in\left(-\infty, \lambda_{1}\right), s_{2} \in\left(\lambda_{1}, \lambda_{2}\right), \ldots, s_{d} \in\left(\lambda_{d-1}, \lambda_{d}\right), s_{d+1} \in\left(\lambda_{d}, 0\right)
$$

such that $H\left(s_{1}\right)=0, \ldots, H\left(s_{d+1}\right)=0$. Since $H$ is a polynomial of degree $d+1$, it does not have other roots. By (6), it is known that $\lambda_{(d+1) / 2}=-1$, so $H(-1) \neq 0$ and therefore, $s_{1} \neq-1, \ldots, s_{d+1} \neq-1$.

Remark 2 Theorem 2 with $d$ odd can be now deduced from Theorems 3 and 5 .

\section{References}

1. Aldroubi, A., Sun, Q., Tang, W-S.: Convolution, average sampling, and a Calderon resolution of the identity for shift-invariant spaces. J. Fourier Anal. Appl. 11, 215-244 (2005)

2. Ericsson, S.: Generalized sampling in shift invariant spaces with frames. Acta Math. Sin. 28, 1823-1844 (2012)

3. García, A., Pérez Villalón, G.: Approximation from shift-invariant spaces by generalized sampling formulas. Appl. Comput. Harmon. Anal. 24, 58-69 (2008) 
4. Kang, S., Kwon, K.H.: Generalized average sampling in shift invariant spaces. J. Math. Anal. Appl. 377, 70-78 (2011)

5. Pérez Villalón, G., Portal, A.: Reconstruction of splines from local average samples. Appl. Math. Lett. 25, 1315-1319 (2012)

6. Pérez Villalón, G., Portal, A.: Sampling in shift-invariant spaces of functions with polynomial growth. Appl. Anal. 92, 2536-2546 (2013)

7. Ponnaian, D., Shanmugam, Y.: Existence and uniqueness of spline reconstruction from local weighted average samples. Rend. Circ. Mat. Palermo 63, 97-108 (2014)

8. Ponnaian, D., Shanmugam, Y.: On the zeros of the generalized Euler-Frobenius Laurent polynomial and reconstruction of cardinal splines of polynomial growth from local average samples. J. Math. Anal. Appl. 432, 983-993 (2015)

9. Schoenberg, I.J.: Cardinal interpolation and spline functions, II: interpolation of data of power growth. J. Approx. Theory 6, 404-420 (1972)

10. Schoenberg, I.J.: Cardinal interpolation and spline functions, IV: the exponential Euler splines. Int. Ser. Numer. Math. 20, 382-404 (1972)

11. Sun, W., Zhou, X.: Average sampling in spline subspaces. Appl. Math. Lett. 15, 233-237 (2002)

12. Sun, W., Zhou, X.: Average sampling in shift invariant subspaces with symmetric averaging functions. J. Math. Anal. Appl. 287, 279-295 (2003)

13. Unser, M., Aldroubi, A.: A general sampling theory for nonideal acquisition devices. IEEE Trans. Signal Process. 42, 2915-2925 (1994) 